\title{
The prospective of Artificial Intelligence in COVID-19 Pandemic
}

\author{
Swati Swayamsiddha ${ }^{1} \cdot$ Kumar Prashant $^{2} \cdot$ Devansh Shaw $^{2} \cdot$ Chandana Mohanty $^{3}$
}

Received: 7 June 2021 / Accepted: 29 August 2021 / Published online: 28 September 2021

(c) IUPESM and Springer-Verlag GmbH Germany, part of Springer Nature 2021

\begin{abstract}
Coronavirus disease 2019 (COVID-19) is a major threat throughout the world. The latest advancements in the field of computational techniques based on Artificial Intelligence (AI), Machine Learning (ML) and Big Data can help in detecting, monitoring and forecasting the severity of the COVID-19 pandemic. We aim to review the detection of the COVID-19 pandemic empowered by AI, major implications, challenges and the future of smart health care at a glance. The AI plays a pioneering role in rapid and improved detection of the disease. It helps in modeling the disease activity and predicting the severity for better decision making and preparedness by healthcare authorities and policymakers. It is a promising technology for automatic and fully transparent monitoring system to track and treat the patients remotely without spreading the virus to others. The future application areas of AI-based healthcare are also identified. The role of AI in tackling the COVID-19 pandemic is reviewed in this paper. AI proves beneficial in early detection with improved results. It also provides solution for contact tracing, prediction, drug development thus reducing the workload of medical industry.
\end{abstract}

Keywords Coronavirus · SARS-CoV-2 · COVID-19 · Artificial Intelligence

\section{Introduction}

The recent 2019 novel coronavirus disease (COVID-19) caused by the infection of severe acute respiratory syndrome Coronavirus 2 (SARS-CoV-2) has emerged as a major public health issue worldwide [1]. The epidemic started with patients reporting pneumonia-like symptoms of unknown etiology, in Wuhan, China (Hubei Province), and has been declared a pandemic by the World Health Organization (WHO) on March 11, 2020 [2]. To date, it has been spotted in over and above 200 countries, infecting more than 171 million people and caused approximately 3.5 million deaths [3]. SARS-CoV-2 is an enveloped RNA virus belongs to the Betacoronavirus group, Sarbecovirus subgenus of Coronaviridae family. Spike glycoproteins cover the outer surface

This article is part of the COVID-19 Health Technology: Design, Regulation, Management, Assessment

Chandana Mohanty

chandanamohanty@gmail.com

1 School of Electronics Engineering, KIIT University, Bhubaneswar, Odisha, India

2 School of Computer Science, KIIT University, Bhubaneswar, Odisha, India

3 School of Applied Science, KIIT University, Bhubaneswar, Odisha, India of CoV-2 thus creating a look of a crown, therefore named corona (Corona means crown in Latin). It shares the same group of 2003 SARS-CoV and hence named SARS-CoV-2. It is the third novel Coronavirus $(\mathrm{CoV})$ that developed into a pandemic in the twenty-first century following the SARS$\mathrm{CoV}$ in 2003 and thereafter the Middle East Respiratory Syndrome Coronavirus (MERS-CoV) in 2012. Since the report of its very first case, the genomic sequence of SARSCoV-2 has mutated and evolved over the years. Scientists and researchers now believe that these changes have augmented the virulence of the virus as a result of which no specific antiviral treatments or vaccines are available for COVID-19 at present [4]. Treatments are mainly based on the management of symptomatic and respiratory support as stated by the respective health authorities of the individual country, where many countries are sticking to protocols issued by WHO [5]. COVID-19 disease has brought the world to a complete standstill affecting the lives of thousands. This global pandemic has spread very rapidly and has tumbled the global economy [1, 4].

The latest advancements in the field of computational techniques, information and communication technologies (ICTs), Artificial Intelligence (AI) and Big Data can extensively help in handling the huge, unprecedented amount of data derived from public health surveillance and real-time epidemic outbreak monitoring and forecasting systems and 
will also provide an option of instant regular situation briefing and updating by governmental institutions and organizations as well as better health resources utilization $[2,6]$. Recently, many companies are using AI to fight COVID-19 namely Deargen, Insilico Medicine, Benevolent AI, SRI Biosciences, and Iktos [7]. Moreover, deep learning (DL) models can predict old and new drugs that might successfully treat COVID-19 [8]. It is indeed a powerful tool to help the world to fight against the COVID-19 pandemic. Thus, the article intends to review the scope of AI to combat against COVID-19 pandemic and also summarize recent prospects and challenges in this upcoming field.

\section{Brief on COVID-19}

SARS-CoV-2 are zoonotic pathogens that originate from animals and can be transmitted to humans through direct contact. Nevertheless, in the majority of cases, these viruses have been reported to be transmitted to humans through an intermediate animal host. It is believed that $\mathrm{CoVs}$ that caused epidemics including COVID-19 have originated from bats $[4,9]$. Bats are the primary hosts of most CoVs. These viruses can cause gastrointestinal illnesses in animals and respiratory illnesses in humans. Typical CoV symptoms include runny nose, cold, cough, sore throat, headache fever, and fatigue to name a few. Hemoptysis and diarrhoea have also been observed in patients with severe illness [10]. However, in immune-compromised patients, there is a chance that these viruses could aggravate and trigger lower respiratory illnesses like pneumonia and bronchitis causing Acute Lung Injury and Acute Respiratory Distress Syndrome which leads to pulmonary failure and ultimately results in a fatality $[4,9]$.

In regards to the structure of CoVs, these are small in size and belongs to the order of Nidovirales, family Coronaviridae, and subfamily Coronavirinae. These are a group of enveloped, positive-sense single-stranded RNA Viruses $[1,10]$. Their diameter size ranges $65-125 \mathrm{~nm}$ and length varies from 26 to 32 kilobases. It has been reported that CoVs carry the largest genomes for RNA viruses. CoVs have been classified and categorized into four major groups based on their genetic and antigenic criteria. These are $\alpha-\mathrm{CoVs}$, $\beta$-CoVs, $\gamma$-CoVs, and $\delta$-CoVs. The $\gamma$-CoVs and $\delta$-CoVs infect birds and might infect mammals, but never been reported to cause any illnesses in humans. On the contrary, $\alpha$-CoVs and $\beta$-CoVs are capable of causing respiratory illnesses in humans and gastrointestinal illnesses in animals $[4,9]$. The genome of CoVs encodes four main fundamental proteins: the spike protein (SP), nucleocapsid protein (NP), membrane protein (MP), and the envelope protein (EP) that are essentially involved in the formation of structurally complete CoVs particle (Fig. 1). The S protein facilitates

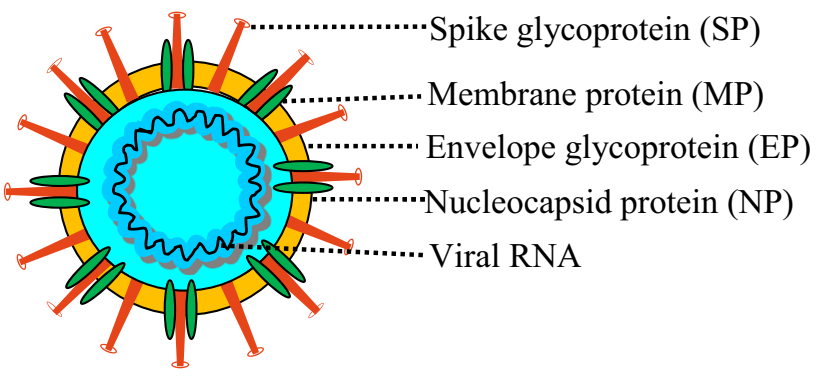

Fig. 1 Schematic figure of the SARS-CoV-2. The SP, MP, and NP are the viral surface proteins that are embedded in a lipid bilayer envelope (EP). The genome is a single-stranded positive-sense viral RNA that is associated with the nucleocapsid protein (NP)

attachment of the virus to the host cell surface receptors angiotensin-converting enzyme-2 (ACE2) that mediates the endocytosis of the virus into the host cell [4]. After internalization inside the host cell the ssRNA $(+)$ genome of $\mathrm{CoVs}$ release into the cytoplasm. The ssRNA $(+)$ genome makes the dsRNA genome which is then transcribed/replicated to form viral mRNAs/new ssRNA $(+)$ genomes. The subgenomic mRNAs translates the major structural proteins. In this regard, the formation of viral nucleocapsids proteins in the cytoplasm takes place and then followed by the assembly and budding into the lumen of the endoplasmic reticulum and Golgi intermediate compartments. Ultimately the assembled mature virion is released from the infected host cell by the process of exocytosis [11]. The life cycle and multiplication of SARS-CoVs inside the infected host cell is illustrated in Fig. 2.

\section{General procedure to identify the COVID-19 symptoms}

There are two broad categories of SARS-CoV-2 identification procedures that were followed and applied for the rapid monitoring of the pandemic disease [12]. One is a conventional laboratory-based testing procedure and the other is AI-based application (Fig. 3). In the former case, the symptomatic persons with influenza-like illness were categorized from the quarantine centre. At the facility level, the symptomatic patients were generally tested with rapid antibody tests[13]. This test identifies the presence of the virus but does not fully confirm if the person is positive. Subsequently, the suspected person or negative COVID-19 persons further tested by real-time reverse transcriptionpolymerase chain reaction (RT-PCR) using throat/nasal swab [9]. Sometimes, patients are subjected to stay under quarantine for about 10 days followed by an antibody test again without the RT-PCR test. Similarly, the COVID-19 
Fig. 2 Schematic diagram showing the replicating cycle of SARS-CoVs-2

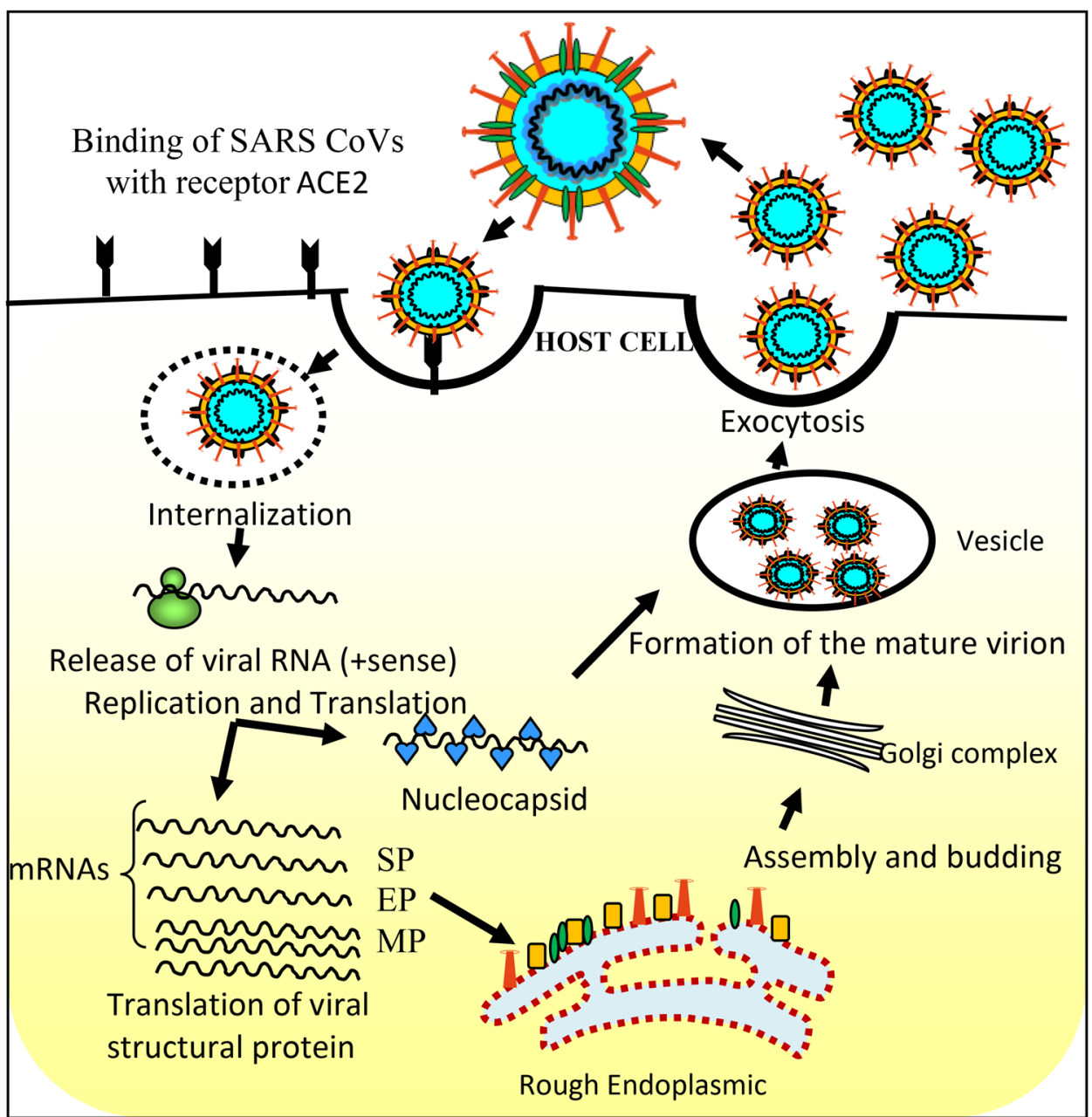

positive patients are given hospital assessment and treatment or isolation as per protocol [9, 12].

The conventional testing procedure based on the RTPCR test is becoming difficult as it is dependent on the preparation of samples and quality control measures. The encountered false-negative RT-PCR test is another hindrance and professionals confirm more than the new coronavirus variant(s), faulty swab sample collection by untrained staff and wrong timing of testing is likely to be the main reasons [14]. Thus, besides conventional testing, other technologies like the use of AI-empowered analysis techniques including image segmentation, classification, and forecasting can result in improved diagnosis of the COVID-19 cases. The AI-based testing procedure mainly focuses on medical imaging such as X-ray and Computed Tomography (CT), Radiology, and predictive analysis procedure. The automatic image acquisition and remote scanning help the lab technicians to perform their jobs with minimal contact with the suspects. After the contact-less image acquisition, the image is segmented to detect the infection in the lungs which help in quantitative analysis and diagnosis of COVID-19. The DL models such as UNet, UNet ++ , VB-Net are used for image segmentation [6]. Further, the severity of the disease is also predicted and the AI-aided treatment and monitoring start for the quarantined patient. During the recovery phase, the above procedure is followed for retests until the patient tests negative. The computer-aided tools help the clinicians and medical specialists to take quick decisions improving their work efficiency and reducing workload [15].

\section{Implication of Al for detecting COVID-19}

AI is something that is considered to revolutionize this world making our work easier, be it from serving food to doing complex works like full automation of industrial works without any human intervention. AI is an approach to make the machines take the decisions on their own with the help of economics, statistical methods, computational intelligence, and traditional coding. It's basically the thinking, learning, working, and decision-making capabilities that a system learns from a human's approach to a problem. It is believed 


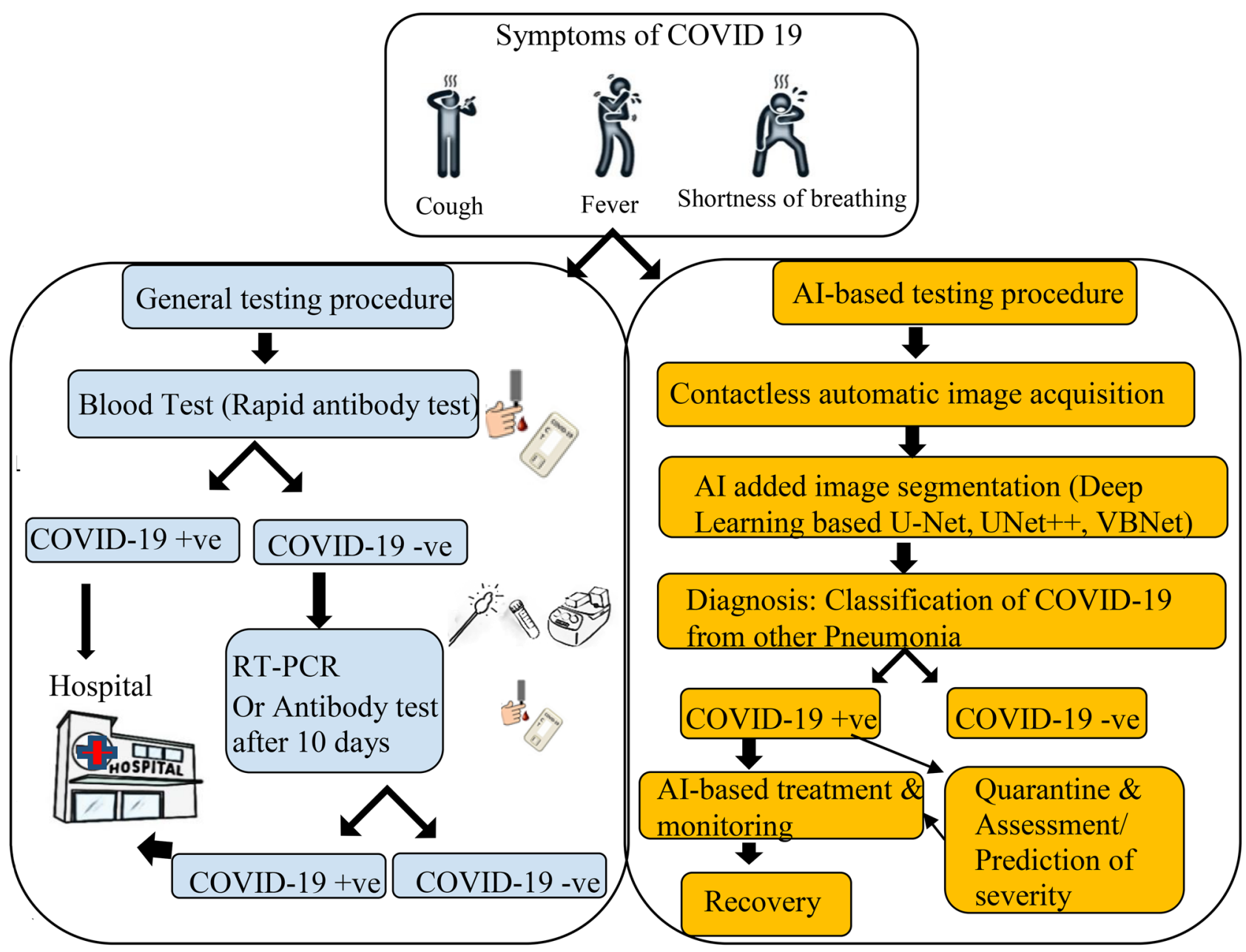

Fig. 3 Schematic diagram showing the conventional general procedure and AI-based applications that generally followed by clinicians to detect the COVID-19 patients

that AI has a solution to every problem. It is one of the most growing fields with a lot of challenges and opportunities [2]. AI is not only making our work easy but also able to work within a limited time constraint. The following sections elucidate the major applications of AI that can contribute to combating against COVID-19 pandemic and the major points listed in Table $1[2,12,15]$.

\section{Diagnosis, treatment, and prognosis}

For diagnosis of COVID-19, a SARS-CoV-2 virus-specific RT-PCR test is routinely used but it takes a maximum of up to two days to give the result, and often serial testing might be necessary to rule out the false results. In today's date, there is a heavy shortage of PCR test kits. Chest CT is a valuable component in the evaluation of patients with suspected SARS-CoV-2 infection [6]. Nevertheless, CT alone may have limited negative predictive value for ruling out SARS-CoV-2 infection, as some patients may have normal radiological findings at the early stages of the disease but, might be corona infected. The AI techniques integrated with the conventional diagnosis can provide robust solution during the screening process $[16,17]$. The thermal face scanners, analysis of medical imaging reports by AI-enabled tools and COVID-19 voice detection platforms are the different ways in which AI is revolutionising COVID-19 screening and diagnosis. We can use AI algorithms to get the dataset of clinical symptoms, exposure history, and laboratory testing to rapidly diagnose patients who are positive of COVID-19 infection. This way we can reduce the workload on our overworked medical staff [15]. We can make use of various modules of AI like machine learning (ML), data science, TensorFlow, neural networks, and various other techniques to help our medical experts in taking accurate decisions using CT scan images. As this method is quite fast and accurate, the results can be obtained in seconds, it can be very useful in diagnosing corona infected patients [18]. 
Table 1 Application areas of AI to combat COVID-19

$\mathrm{AI}$ and its implications in different area

Diagnosis and treatment

COVID-19 genome analysis

COVID-19 vaccine development

Infection tracking

Prediction of patient outcome

Computational biology and medicines perspective

Protein structure predictions

Drug discovery and digital health

Awareness and social control through the Internet

Reducing the workload of the medical staffs
Status/report of applications

The AI-based testing procedure mainly focuses on medical imaging such as X-ray and computed tomography (CT), radiology, and predictive analysis procedure. The deep learning models such as UNet, UNet ++ , VB-Net are used for image segmentation, classification, and assessing the severity of the disease with high accuracy. AI4COVID-19 app helps in preliminary diagnosis using cough samples. Bluedot and InferVision are the medical AI-based enterprises, help in early detection of COVID-19

Genome sequencing of hCOV-19 is available via Global Initiative on Sharing All Influenza Data (GISAID) enabling rapid and open access to virus data

Using the Vaxign reverse vaccinology-machine learning platform, suitable vaccine candidates can be predicted and COVID-19 High Performance Computing (HPC) Consortium is engaged in providing advanced computing resources for such projects

The abnormal respiration pattern classifier based on Respiratory Simulation Model can help in large scale screening and tracking of COVID-19 patients. The ML-based prediction model deployed using the FogBus framework can predict the growth and trend of the pandemic. HealthMap helps in tracking and monitoring COVID-19 spread by gathering data in daily basis

XGBoost based machine learning platform can predict the survival rate of highly critical COVID-19 patients. EpiRisk is another such tool used for prediction of infection

Baricitinib is identified by Benevolent AI's knowledge graph as a potential drug to combat COVID-19

Google's AI platform DeepMind-based protein structure prediction tool AlphaFold has predicted and released the 3D structures of several understudied proteins of the CoV-2 as an open-source. Antivirals/ vaccines can be designed to combat the forthcoming COVID-19 pandemic. DeepTracer, a DNN based software can predict protein structure of SARS-CoV-2

Structure-based virtual screening for drug discovery and drug repurposing. Data mining, machine learning, high-level quantum mechanical (QM), quantum- mechanical/ molecular-mechanical (QM/MM), and quantitative structure-activity relationship (QSAR) techniques are useful to accelerate the drug discovery program. AI-based inclProject IDentif.AI and PolypharmDB helps in

identifying drugs against COVID-19

AI-enabled social media (Facebook and Twitter) survey enables monitoring and forecasting the spread of COVID-19 and thus helps in better preparedness and control. The range of apps like Arogya Setu(India), CloseContact (China), HaMagen (Israel), Mawid (Saudi Arabia), Tabaud (Saudi Arabia), Tawakkalna (Saudi Arabia), Sehha (Saudi Arabia), TraceTogether (Singapore), Covid Safe(Australia), Immuni(Italy), COVID Symptom Study(UK), NHS COVID-19(UK), COVID Watch (USA) \& PathCheck SafePlaces (USA)

gives information about the vicinity of a corona positive patient, risk assessment, test reports, educational resources, personalized health services and vaccination information

The COVID-19 related inquiries of the public are addressed by medical 'chatbots' like Zini and Clara from the Centre for Disease Control. CovNet is developed to extract visual features from a CT scan predicting the contamination. CRUZR robot assist in high exposure tasks at hospitals
References

$[24,32,48-50]$

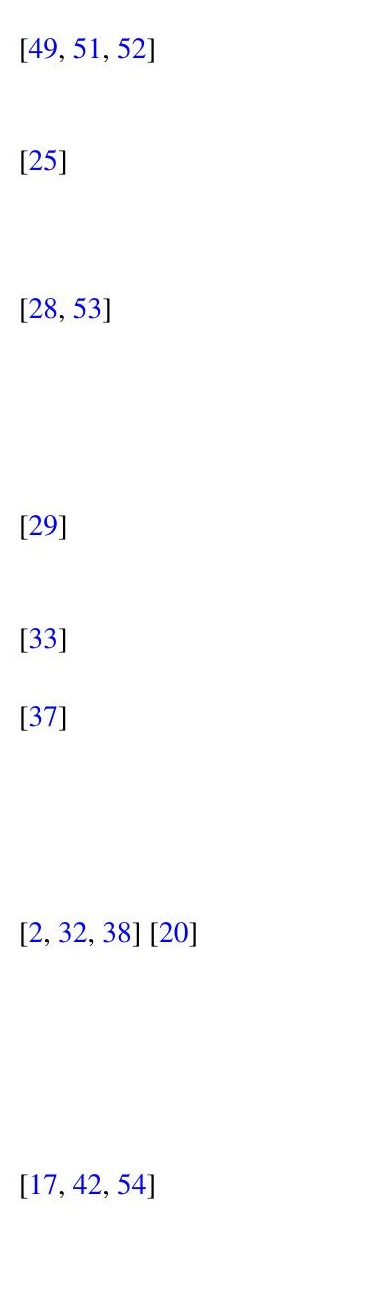

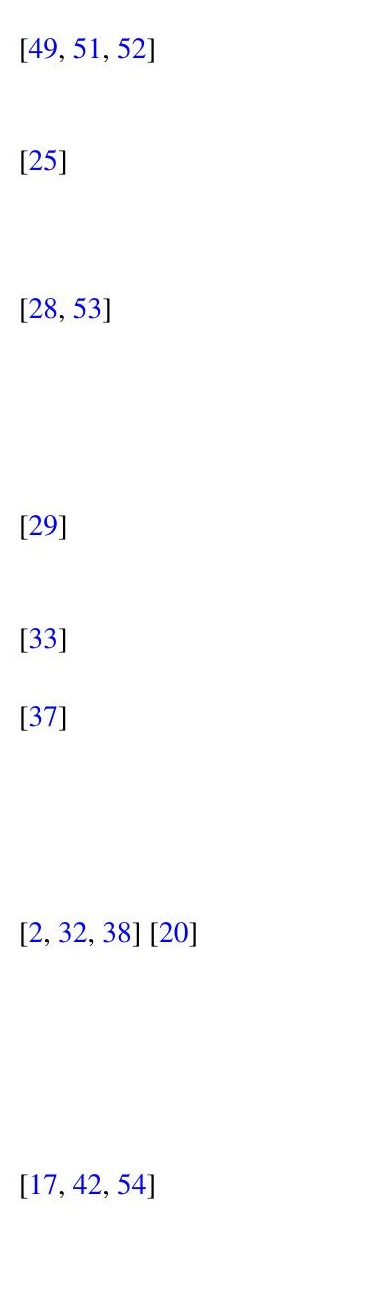

$[49,51,52]$
$[25]$
$[28,53]$
$[29]$
$[33]$
$[37]$
$[2,32,38][20]$
$[17,42,54]$
$[20]$

(1)

$[49,51,52]$
$[25]$
$[28,53]$
$[29]$
$[33]$
$[37]$

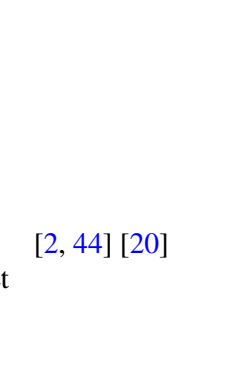

\section{COVID-19 genome analysis}

The process of identifying the sequence of a genome is known as Genome Sequencing. The size of SARS-CoV-2 genome is of approx. 30 kilobyte. AI techniques have been potentially used to retrieve interesting information from COVID-19 genome sequences. The field of pattern analysis provides effective computer-based tools that allow humans, particularly bioinformaticians, to exam- 
ine complicated and huge genetic and genomic data sets. Sequential pattern mining (SPM) is a technique of Data Mining that is used on computer understandable corpus of COVID-19 genome sequences to look for the hidden patterns which reveals frequent patterns of nucleotide bases and their relationships with each other [19]. Various sequence prediction models are applied on the obtained corpus so as to evaluate if any nucleotide base(s) can be predicted. Then further algorithms are designed such that they can accurately find the change in nucleotide base positions so that the mutation rate can be predicted and if possible the next variants can be predicted. For mutation analysis in genome sequences, algorithm have been designed to find the locations in the genome sequences where the nucleotide bases are changed and to calculate the mutation rate. Thus, SPM data can effectively disclose new information on virus mutations, strains, pathogenicity, and clinical symptoms $[19,20]$. For instance, the dynamics of genome sequencing of different variants of hCOV-19 is available via Global Initiative on Sharing All Influenza Data (GISAID) enabling rapid and open access to pandemic virus data. It is an initiative in which tens of thousands of COVID-19 virus samples taken from patients around the world and have been genetically sequenced and analyzed using $\mathrm{AI}$ algorithms to conquer COVID-19 [21].

\section{COVID-19 vaccine development}

AI has proven to have a revolutionary impact in the development of Covid-19 vaccine by predicting probable epitopes which might have the prime antigenic property. AI methods have been successfully assist tackle new COVID-19 mutations. Some of the machine learning approaches which helps in identifying the protein sequences are Support Vector Machine, Random Forest, Recursive Feature Selection but they have low efficiency in predicting the epitopes so more advanced tools like Deep Convolutional Neural Networks are being used for this purpose. Some of the supervised neural network algorithms such as MARIA [22] and NetMHCPan4 [23] and Linear Regression Model known as Disco Tope, suggested a number of probable epitopes which can be easily recognized by the $\mathrm{T}$ cells. Using the Vaxign reverse vaccinology-machine learning platform. SARS-CoV-2 has a structural spike protein (SP) and five non-structural proteins (nsp3, 3CL-pro, and nsp8-10). These proteins has crucial role for viral adhesion and consequence host invasion. These proteins can be predicted by Vaxign and the newly developed machine learning-based VaxignML reverse vaccinology tools to predict COVID-19 vaccine candidates. In similar approach, Ong et al. used the Vaxign-ML reverse vaccinology technology, which relied on supervised classification models, to forecast potential vaccine candidates for COVID-19 [20]. AI has been play- ing a major role since the outbreak of the pandemic and is now helping in vaccination by determining the specific age group as well as the region, thus enhancing the decision making capability of humans. It is playing a major role in the vaccine supply chain reducing the risk factors, enables predictions of revenue loss, and keeps a track of the supplies delivered as well as supplies available in inventory, monitoring the temperature control of the vaccines and continuous analyzing of the real-time data [24]. The analyzing of real-time data with previously collected data and models enables to predict the next specific targets for vaccination. In order to provide computing power for learning and validating vaccine response models, to accelerate understanding and modelling of the patient's response and evaluate the therapies, the COVID-19 High Performance Computing (HPC) Consortium is engaged in providing advanced computing resources such as powerful data analytics, machine learning and AI [25].

\section{Infection tracking and prediction}

$\mathrm{CoV}$ has already done a hugely destructive impact on mankind and the world economy, and now it's of urgent need to bring this under control. Till now the only way is to "Track And Quarantine" and this is where AI can be very useful. AI can keep track of the persons who came in close contact with the infected person and the data available can be used to quarantine the suspected contaminants $[9,26]$. Contact tracing is a process that is used to identify, educate, and monitor individuals who have had close contact with someone who is infected with the virus. These individuals are at a greater risk of becoming infected with the deadly $\mathrm{CoV}$ and with the help of AI we can protect them by contact tracing the persons who have come in contact with the recently infected persons and thereby limiting the speed of the spread of the virus [27].

\section{$V$. Prediction of patient outcome}

$\mathrm{CoV}$ has attracted the attention of every field of science and AI is not an exception to this. Researchers are trying to find out ways in which they can mitigate and easily track the spread of this virus and their AI took the place because it has been found from the studies that using technologies like AI we can identify the high-risk patients, providing the best medical help, proper screening, controlling their spread in real-time, the future course of the disease and many more. Doctors are also looking for new automatic and fully transparent monitoring systems to track and treat the patients and AI is expected to fill this need $[28,29]$. AI can learn from records of patients and can predict better planning, medical facilities, and the future spread of this virus.

\section{Medicines perspective and repurposing}

AI can link the datasets which a human brain can take years to do, hence it can be used for finding the correct combination of drugs that would be most effective in combating the 
COVID-19 disease [9]. The practice of an existing drug to treat a new disease is called Drug Repurposing [30]. It is the best, fastest, and quickest hope against COVID-19. Recently, The AI system recognized approximately 80 marketed drugs that presume to be effective against SARSCoV-2 [31]. Drugs like bedaquiline, brequinar, celecoxib, clofazimine, conivaptan, gemcitabine, tolcapone, and vismodegib have shown potent in vitro activities against the proliferation of feline infectious peritonitis (FIP) virus in Fcwf-4 cells. The most potent existing drugs like boceprevir, chloroquine, homoharringtonine, tilorone, and salinomycin were found potent during the workouts of $\mathrm{AI}$ methods [32]. In this regard, we can have computing simulations to speed up the ability to screen billions of existing drugs for their interactions with and ability to disrupt SARS-CoV-2 proteins. Then the shortlisted drugs can be put into the trial to check which is most effective on the viral proteins and then can be used on the patients for better treatment and quick recovery [33].

\section{Protein structure prediction}

At the time of viral infection, the virus enters into the host cell and interacts with its protein-making machinery, utilizing it to produce a group of proteins that can copy the viral RNA molecule. These RNA-duplicating proteins are known as polymerase, an alluring target for viral infection. By inhibiting these RNA polymerases, the virus cannot reproduce thereby stopping an infection [34]. Protein structure prediction can be done by two methods: template modeling and template-free modeling. The former one predicts the structure of a protein by utilizing similar proteins as a template sequence and the latter one predicts structure for proteins that have unknown related structures $[35,36]$. AlphaFold algorithm predicts diversity of membrane protein structures linked with $\mathrm{CoV} 2$ like protein $3 \mathrm{a}$, nsp2, nsp4, nsp6, and papain-like Cterminal domain. The prediction about the protein structure and its ducking site could open up new drug discovery to combat COVID-19 [37].

\section{Drug discovery and digital health}

$\mathrm{AI}$ is an incredible tool used by researchers to discover new drugs, for disease diagnosis and prediction thereby promote digital health technology to manage CoVs related public health challenges. The verity of data related to CoVs has been analyzed immensely by various researchers with the aid of AI, ML, and DL [24, 36]. Quick identification of drugs that could target to CoVs was now feasible with the assistance of AI and ML [35, 36]. Furthermore, this technology rampantly used to narrow down in selecting the best target drug among numerous to fasten the clinical study. In a view to targeting CoVs, AI has accelerated the new drug discovery procedure and recently reported its novel intervention to select possible target drugs for repurposing [38]. In this regard, Beck et al. and Stebbing et al. have potentially repurposed the drug atazanavir and Baricitinib respectively in a separate study using Machine Learning to treat COVID-19 [39, 40]. AI platform can be used to study the three-dimensional structure of the drug, its specificity to predict chemical interactions with a potential target. Currently, the synergy of COVID-19 related health information, drug discoveries, and digital technologies play a crucial role to tackle the COVID-19 pandemic. The collection of databases through Electronic Health Records and the Internet of Things (IoT) has led to a demand for big data analytics and $\mathrm{AI}$ integration in healthcare services [5]. Thus, current Healthcare IT News/ HIMSS Analytics survey, healthcare organizations confirmed that AIs play a substantial role in the areas of population health, clinical decision support, patient diagnosis and precision medicine $[2,39,40]$.

IX. Awareness and social control through the Internet The live updates about the status of the COVID-19 cases are reported through official websites as well as through digital media platforms. The precautionary measures and helpline numbers are broadcast through social media by authentic government accounts [41]. The public is made aware of the various initiatives of policymakers through the internet and phone calls. AI plays a crucial role to identify and delete the fake news circulated on social media. The AI-empowered apps warns the individual about the proximity of COVID-19 case if it is within the vicinity of the person. Also, a person can self-assess his/her health condition by answering certain questions put forward by the AI-empowered app. This GPS and Bluetooth enabled app such as Arogya Setu (India), Close Contact (China) and many new app have mentioned in Table 1 that helps to track the COVID-19 infection and makes an individual aware of real-time data [42]. The smartphone thermometer also helps in self-assessing the level of fever of an infected person. Also, AI-aided cough type detection by audio call recording is helpful to detect symptomatic cases [43].

\section{$\mathrm{X}$. Reducing the workload of the medical staffs}

The healthcare professionals are in total burnout with this sudden surge in the number of cases of novel $\mathrm{CoV}$ as it has increased the challenges for them to keep themselves safe and treat the patients. AI has successfully enhanced the image processing techniques by designing the chest X-ray system which can auto-scan the $\mathrm{CoV}$ patient's lungs within a few seconds thus reducing doctor's intervention. An AIbased deep learning model named COVID-19 detection neural network (COVNet) is also developed to extract visual features from $\mathrm{CT}$ scan of $\mathrm{CoV}$ patients thus predicting the contamination. In this way, $\mathrm{AI}$ is helping healthcare professionals to do their job seamlessly and providing them with more smart tools to deal with this pandemic [2, 44]. 


\section{Future opportunity and challenges at a glance}

As we all know the CoV can spread from human to human so medical staff is at high risk of being infected but medical robots are preventable to cross infections and this can be revolutionary in cases of viral outbreaks. The world has witnessed one case in the US already where a man who was infected with the $\mathrm{CoV}$ was being treated by a robot [2]. The robot has a visual screen like TV which allows physicians to communicate with the patients and it is also equipped with stethoscope helping doctors take the man's health report while minimizing exposure of medical staffs to the patients and of course patients who are in quarantine could be treated better with medical robots. Further drones can also play a vital role in stopping the outbreak of this infection by supporting the medical deliveries in quarantine zones as they can safely collect the medicine and deliver it to people who are in need [29]. In the last decade, AI has gained remarkable momentum in smart healthcare and if harnessed in the right way, it can contribute to efficiently fighting the COVID-19 pandemic. On the other hand, along with positive contributions, many challenges need to be worked on [45]. The application of AI in COVID-19 diagnosis is in the nascent stage. In DL, numerous and quality data sets of chest X-ray or CT scan are required for training the system. But, the systematic collection of these data sets is difficult in this pandemic situation. The automatic image acquisition system is to be more precise to capture high-quality images with reduced radiation exposure. Moreover, it needs AI-literate lab technicians to handle the case. Though AI-based diagnosis is faster and safe, it gives partial information about COVID-19 patients and may give negative results in the early stage, so the clinical testing methods need to be integrated for better detection and diagnosis of COVID-19 patients [6]. Also, there are ethical, social and human rights challenges of AI in public health. The diagnostic data sharing needs consent of the involved population and trust towards different entities involved. Since AI is mainly data-driven, the privacy and genuineness of the data sets can be challenging. For example, in India assigning a unique identification code to every individual and linking it for medical records and relating it for health benefits pose a social and legal challenge. The challenges of privacy, transparency, consent, and trust need to be managed by consensus-based guidelines [46]. Another important challenge is that AI-based techniques such as Artificial Neural Network, ML and DL are considered as 'blackbox', hence the internal parameters cannot be tuned rather only the outputs are obtained according to the input datasets. Hence, it is difficult to translate highly sensitive AI models according to the clinical situation. Also, the efficacy, fairness and stability of the AI models need to tested rigorously to ensure its validity before the actual deployment. Adequate availability of the data sets for training and validation is an inherent limitation of the AI-based models. Another challenge is that even though the AI based design programs are powerful functions, these are vulnerable to security attacks which can lead to error and hamper the health of the patient. AI-based contact tracing and surveillance system also raises the privacy issues. Hence, responsible deployment of the AIbased techniques and robust modeling can help to overcome such limitations [20, 47].

\section{Conclusion}

The pandemic of COVID-19 has crumbled the public healthcare system and has become a global threat. This paper surveys the potential applications of $\mathrm{AI}$ in fighting the COVID-19. The AI-aided detection is safe, accurate, and faster compared to the conventional procedure. The overall efficiency of medical professionals is increased with AI-empowered diagnosis, tracking, and treatment of the patients. The implications of AI in tackling COVID-19 are identified which include protein structure prediction, monitoring of the treatment, awareness and social control, development of drugs, and digital health. The AI-enabled imaging gives partial information about the COVID-19 which should be integrated with the laboratory examination for better diagnosis and screening. In this scenario, AI can assist in integrating the data from both sources and provide rapid and more accurate results. Thus, AI proves as an innovative technology for fighting against the COVID-19 pandemic and can revolutionize the smart-health care industry in near future.

Acknowledgements Chandana Mohanty would like to thank Department of Science and Technology (DST), Government of India, for the award No.SR/WOS-A/LS-1405/2015.

\section{Declarations}

Informed consent The authors confirm that this article content has no conflict of interest. I further confirm that this article is original and has not been published elsewhere nor is it currently under consideration for publication elsewhere

\section{Conflict of interest}

- All authors have participated in (a) conception and design, or analysis and interpretation of the data; (b) drafting the article or revising it critically for important intellectual content; and (c) approval of the final version.

- This manuscript has not been submitted to, nor is under review at, another journal or other publishing venue.

- The authors have no affiliation with any organization with a direct or indirect financial interest in the subject matter discussed in the manuscript 
- The following authors have affiliations with organizations with direct or indirect financial interest in the subject matter discussed in the manuscript:

Research involving human participants and/or animals NA

\section{References}

1. Haleem A, Javaid M, Vaishya R. Effects of COVID-19 pandemic in daily life. Curr Med Res Pract. 2020;10(2):78-9. https://doi. org/10.1016/j.cmrp.2020.03.011.

2. Ting DSW, Carin L, Dzau V, Wong TY. Digital technology and COVID-19. Nat Med. 2020;26(4):459-61. https://doi.org/10. 1038/s41591-020-0824-5.

3. World meter hwwic. 2020 May 8.

4. Di Wu, Tiantian Wu, Liu Q. The SARS-CoV-2 outbreak: What we know. Int J Infect Dis 2020;94(2):44-8.

5. Swayamsiddha S, Mohanty C. Application of cognitive Internet of Medical Things for COVID-19 pandemic. Diabetes Metab Syndr. 2020;14(5):911-5. https://doi.org/10.1016/j.dsx.2020.06.014.

6. Shi F, Wang J, Shi J, Wu Z, Wang Q, Tang Z et al. Review of artificial intelligence techniques in imaging data acquisition, segmentation and diagnosis for covid-19. . IEEE Reviews in Biomedical Engineering 2020 2020;2(1):220-35.

7. IEEE Spectrum FCUAtFC. https://spectrum.ieee.org/the-humanos/artificial-intelligence/medical-ai/companies-ai-coronavirus. 2020.

8. Arrietaa A, Díaz-Rodríguez N, Del J. Explainable Artificial Intelligence (XAI): Concepts, taxonomies, opportunities and challenges toward responsible AI. Information Fusion. 2020;58(4):82-115.

9. Tobaiqy M, Qashqary M, Al-Dahery S. Therapeutic management of patients with COVID-19: a systematic review. Infection Prevention in Practice. 2020;2(3):22-8.

10. Pooladanda V, Thatikonda S, Godugu C. The current understanding and potential therapeutic options to combat COVID-19. Life Sci. 2020;254: 117765. https://doi.org/10.1016/j.lfs.2020.117765.

11. Valencia D. Brief Review on COVID-19: The 2020 Pandemic Caused by SARS-CoV-2 Cureus. 2020;12(3):73-86.

12. Vaishya R, Javaid M, Khan IH, Haleem A. Artificial Intelligence (AI) applications for COVID-19 pandemic. Diabetes Metab Syndr. 2020;14(4):337-9. https://doi.org/10.1016/j.dsx.2020.04.012.

13. Nataranjan D. COVID-19: What Are Serological Tests, and How Can They Help India? Science the wire. 2020:https://science.thewire.in/ the-sciences/icmr-rt-pcr-serological-test-antibodies-blood-plasma/.

14. Afzal A. Molecular diagnostic technologies for COVID-19: Limitations and challenges. J Adv Res. 2020;26:149-59. https://doi. org/10.1016/j.jare.2020.08.002.

15. Mei X, Lee HC, Diao KY, Huang M, Lin B, Liu C et al. Artificial intelligence-enabled rapid diagnosis of patients with COVID-19. Nat Med. 2020;2(5):112*35. https://doi.org/10.1038/ s41591-020-0931-3.

16. Jin C, Chen W, Cao Y, Xu Z, Tan Z, Zhang X, et al. Development and evaluation of an artificial intelligence system for COVID-19 diagnosis. Nat Commun. 2020;11(1):50-88. https://doi.org/10. 1038/s41467-020-18685-1

17. V. Chamola, V. Hassija, Gupta V, M. Guizani. A Comprehensive Review of the COVID-19 Pandemic and the Role of IoT, Drones, AI, Blockchain, and 5G in Managing its Impact. IEEE Access. 2020;8(2):90225-65. https://doi.org/10.1109/ACCESS. 2020.2992341 .
18. Han.Z, Wei. B, Hong.Y. Accurate Screening of COVID-19 using Attention Based Deep 3D Multiple Instance Learning. IEEE Reviews in Biomedical Engineering 2020;2(1):220-35.

19. Nawaz MS, Fournier-Viger P, Shojaee A, Fujita H. Using artificial intelligence techniques for COVID-19 genome analysis. Applied Intelligence. 2021;1-18. doi:https://doi.org/10.1007/ s10489-021-02193-w.

20. Arora N, Banerjee AK, Narasu ML. The role of artificial intelligence in tackling COVID-19. Futur Virol. 2020;15(11):717-24. https://doi.org/10.2217/fvl-2020-0130.

21. Organization. WH. Operational considerations to expedite genomic sequencing component of GISRS surveillance of SARS-CoV-2.https://www.who.int/publications/i/item/WHO2019-nCoV-genomic-sequencing-GISRS-2021.1

22. Chen B, Khodadoust MS, Olsson N, Wagar LE, Fast E, Liu $\mathrm{CL}$, et al. Predicting HLA class II antigen presentation through integrated deep learning. Nat Biotechnol. 2019;37(11):1332-43. https://doi.org/10.1038/s41587-019-0280-2.

23. Jurtz V, Paul S, Andreatta M, Marcatili P, Peters B, Nielsen M. NetMHCpan-4.0: Improved Peptide-MHC Class I Interaction Predictions Integrating Eluted Ligand and Peptide Binding Affinity Data. J Immuno (Baltimore, Md : 1950). 2017;199(9):3360 8. https://doi.org/10.4049/jimmunol.1700893.

24. Ahuja AS, Reddy VP, Marques O. Artificial intelligence and COVID-19: A multidisciplinary approach. Integr Med Res. 2020;9(3): 100434. https://doi.org/10.1016/j.imr.2020.100434.

25. The COVID-19 High Performance Computing Consortium. https://covid19-hpc-consortium.org/. 2021.

26. Lalmuanawma S, Hussain J, Chhakchhuak L. Applications of machine learning and artificial intelligence for Covid-19 (SARSCoV-2) pandemic: A review. Chaos Solitons Fractals. 2020;139: 110059. https://doi.org/10.1016/j.chaos.2020.110059.

27. Srinivasa Rao ASR, Vazquez JA. Identification of COVID-19 Can Be Quicker Through Artificial Intelligence Framework Using a Mobile Phone-Based Survey When Cities and Towns Are Under Quarantine. Infect Control Hosp Epidemiol. 2020;3(1):1-5.

28. Tuli S, Tuli S, Tuli.R. Predicting the growth and trend of COVID19 pandemic using machine learning and cloud computing. Internet of Things. 2020;11(1):110-21.

29. Yan L, Zhang H, J G. A machine learning-based model for survival prediction in patients with severe COVID-19 infection. medRxiv Prepr. 2020.

30. Zhou Y, Wang F, Tang J, Nussinov R, Cheng F. Artificial intelligence in COVID-19 drug repurposing. Lancet Digit Health. 2020;2(12):66776. https://doi.org/10.1016/S2589-7500(20)30192-8.

31. Mohanty S, Harun Ai Rashid M, Mridul M, Mohanty C, Swayamsiddha S. Application of Artificial Intelligence in COVID19 drug repurposing. Diabetes Metab Syndr. 2020;14(5):1027-31. https://doi.org/10.1016/j.dsx.2020.06.068.

32. Ke YY, Peng TT, Yeh TK, Huang WZ, Chang SE, Wu SH et al. Artificial intelligence approach fighting COVID-19 with repurposing drugs. Biomed J. 2020;4(12):52*68. https://doi.org/10. 1016/j.bj.2020.05.001.

33. Richardson.P, Griffin.I., Tucker.C. Baricitinib as Potential Treatment for 2019-nCoV Acute Respiratory Disease. Lancet. 2020;395(5):102-23.

34. Pachetti M, Marini B, Benedetti F, Giudici F, Mauro E, Storici $\mathrm{P}$, et al. Emerging SARS-CoV-2 mutation hot spots include a novel RNA-dependent-RNA polymerase variant. J Transl Med. 2020;18(1):179. https://doi.org/10.1186/s12967-020-02344-6.

35. Hassanzadeh P, Atyabi F, Dinarvand R. The significance of artificial intelligence in drug delivery system design. Adv Drug Deliv Rev. 2019;151-152(4):169-90. https://doi.org/10.1016/j.addr.2019.05.001.

36. Persidis A, Persidis A. Artificial intelligence for drug design. Nat Biotechnol. 1997;15(10):1035-6. https://doi.org/10.1038/nbt1097-1035. 
37. Gulamali F. AlphaFold Algorithm Predicts COVID-19 Protein Structures. InfoQ. 2020;2(4):5-8.

38. Gordon DE, Jang GM, Bouhaddou M, Xu J, Obernier K, White $\mathrm{KM}$, et al. A SARS-CoV-2 protein interaction map reveals targets for drug repurposing. Nature. 2020;12(10):112-25. https://doi. org/10.1038/s41586-020-2286-9.

39. Beck B, Shin B, Choi Y. Predicting commercially available antiviral drugs that may act on the novel coronavirus (SARS-CoV-2) through a drug-target interaction deep learning model. Comput Struct Biotechnol J. 2020;18(9):784-90.

40. Stebbing J, Krishnan V, de Bono S, Ottaviani S, Casalini G, Richardson PJ, et al. Mechanism of baricitinib supports artificial intelligence-predicted testing in COVID-19 patients. EMBO Mol Med. 2020;12(8): e12697. https://doi.org/10.15252/emmm. 202012697.

41. Naudé W. Artificial intelligence vs COVID-19: limitations, constraints and pitfalls. AI \& Soc. 2020;35(3):761-5. https://doi.org/ 10.1007/s00146-020-00978-0.

42. Allam Z, Jones D. On the Coronavirus (COVID-19) Outbreak and the Smart City Network: Universal Data Sharing Standards Coupled With Artificial Intelligence (AI) to Benefit Urban Health Monitoring and Management. Healthcare (Basel). 2020;8(12):32-46.

43. Maddah E, B B. Use of a Smartphone thermometer to monitor thermal conductivity changes in diabetic foot ulcers: A pilot study. J Wound Care. 2020;29(1):61-6.

44. Kumar A, Gupta PK, Srivastava A. A review of modern technologies for tackling COVID-19 pandemic. Diabetes Metab Syndr. 2020;14(4):569-73. https://doi.org/10.1016/j.dsx.2020.05.008.

45. Kaplan A, M. H. Rulers of the world, unite! The challenges and opportunities of artificial intelligence. Business Horizons. 2020;16(1):37-50.

46. Goodman K, Zandi D, Reis AEV. Balancing risks and benefits of artificial intelligence in the health sector. Bulletin of the World Health Organization. 2020;98(4):230-35.
47. Hu Y, Jacob J, Parker GJM, Hawkes DJ, Hurst JR, Stoyanov D. The challenges of deploying artificial intelligence models in a rapidly evolving pandemic. Nature Machine Intelligence. 2020;2(6):298-300. https://doi.org/10.1038/s42256-020-0185-2.

48. Imran A, Posokhova I, Qureshi HN, Masood U, Riaz MS, Ali $\mathrm{K}$, et al. AI4COVID-19: AI enabled preliminary diagnosis for COVID-19 from cough samples via an app. Inform Med Unlocked. 2020;20: 100378. https://doi.org/10.1016/j.imu.2020. 100378.

49. Shamman AH, Hadi AA, Ramul AR, Zahra MMA, Gheni HM. The artificial intelligence (AI) role for tackling against COVID19 pandemic. Mater Today Proc. 2021. https://doi.org/10.1016/j. matpr.2021.07.357.

50. Fong S, Dey N, Chaki J. Artificial Intelligence for Coronavirus Outbreak. Springer; 1st ed 2021 edition. 2021:117.

51. Lalmuanawma S, Hussain J, Chhakchhuak L. Applications of machine learning and artificial intelligence for Covid-19 (SARS-CoV-2) pandemic: A review. Chaos Solitons Fractals. 2020;139(5):110059-70. https://doi.org/10.1016/j.chaos.2020. 110059.

52. Noorden R. Scientists call for fully open sharing of coronavirus genome data. Nature. 2021;3(2):195-6.

53. Wang Y, Hu M, Li Q. Abnormal respiratory patterns classifier may contribute to large-scale screening of people infected with COVID-19 in an accurate and unobtrusive manner. COVID-19 e-print. 2020;1(2):42-57.

54. Alanzi T. A Review of Mobile Applications Available in the App and Google Play Stores Used During the COVID-19 Outbreak. J Multidiscip Healthc. 2021;14:45-57. https://doi.org/10.2147/ jmdh.S285014.

Publisher's Note Springer Nature remains neutral with regard to jurisdictional claims in published maps and institutional affiliations. 